\title{
Coarse-Grained Entanglement and Operator Growth in Anomalous Dynamics
}

\author{
Zongping Gong, ${ }^{1}$ Adam Nahum $\odot^{2,3}$ and Lorenzo Piroli $\circledast^{1,4}$ \\ ${ }^{1}$ Max-Planck-Institut für Quantenoptik, Hans-Kopfermann-Straße 1, D-85748 Garching, Germany \\ ${ }^{2}$ Theoretical Physics, University of Oxford, Parks Road, Oxford OX1 3PU, United Kingdom \\ ${ }^{3}$ Laboratoire de Physique de l'École Normale Supérieure, CNRS, ENS and Université PSL, \\ Sorbonne Université, Université de Paris, 75005 Paris, France \\ ${ }^{4}$ Philippe Meyer Institute, Physics Department, École Normale Supérieure (ENS), Université PSL, \\ 24 rue Lhomond, F-75231 Paris, France
}

(Received 22 September 2021; accepted 31 January 2022; published 23 February 2022)

\begin{abstract}
In two-dimensional Floquet systems, many-body localized dynamics in the bulk may give rise to a chaotic evolution at the one-dimensional edges that is characterized by a nonzero chiral topological index. Such anomalous dynamics is qualitatively different from local-Hamiltonian evolution. Here we show how the presence of a nonzero index affects entanglement generation and the spreading of local operators, focusing on the coarse-grained description of generic systems. We tackle this problem by analyzing exactly solvable models of random quantum cellular automata (QCA) that generalize random circuits. We find that a nonzero index leads to asymmetric butterfly velocities with different diffusive broadening of the light cones and to a modification of the order relations between the butterfly and entanglement velocities. We propose that these results can be understood via a generalization of the recently introduced entanglement membrane theory, by allowing for a spacetime entropy current, which in the case of a generic QCA is fixed by the index. We work out the implications of this current on the entanglement "membrane tension" and show that the results for random QCA are recovered by identifying the topological index with a background velocity for the coarse-grained entanglement dynamics.
\end{abstract}

DOI: 10.1103/PhysRevLett.128.080602

Introduction.-In many-body quantum systems subject to local-Hamiltonian dynamics, correlations propagate with a finite velocity, which determines an approximate causal cone [1]. This property, however, holds beyond local-Hamiltonian evolution, defining a more general class of locality-preserving (LP) unitary dynamics that are termed quantum cellular automata (QCA) whenever the causal cone is strict [2,3]. Models of LP evolution appear naturally at the boundary of certain Floquet systems displaying many-body localization (MBL) in the bulk [4-8] (cf. Fig. 1).

In one dimension (1D), the mathematical theory of LP dynamics is well developed [10-17]. A crucial result, proven in Ref. [11], is that QCA are fully classified by a genuinely dynamical topological index. This result was recently generalized to include the more realistic case where the causal cone is only approximate [18]. Importantly, this topological index is zero if and only if the evolution is generated by a (quasi)local Hamiltonian.

Published by the American Physical Society under the terms of the Creative Commons Attribution 4.0 International license. Further distribution of this work must maintain attribution to the author(s) and the published article's title, journal citation, and DOI. Open access publication funded by the Max Planck Society.

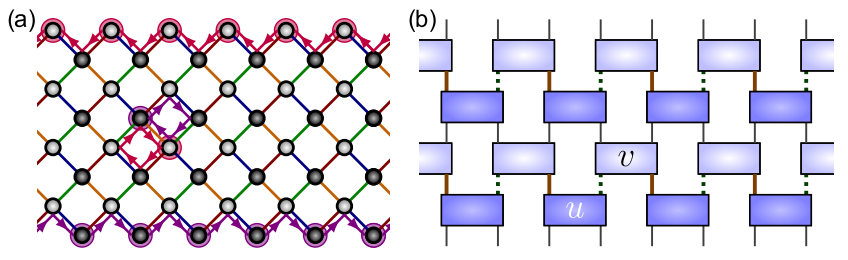

FIG. 1. (a) Pictorial representation of LP evolution as the edge dynamics of a Floquet qudit system. In the presence of a MBL phase in the bulk [9], it is always possible to decompose the oneperiod Floquet operator as [4] $U_{F}=U_{\text {edge }} e^{-i H_{\text {bulk }} T}$, where $H_{\text {bulk }}$ is a MBL Hamiltonian, and $U_{\text {edge }}$ is an effective $1 \mathrm{D}$ evolution acting on qudits within a few localization lengths from the boundary. (b) Any LP evolution $\mathcal{U}(t)$ may be approximated by a QCA in Margolus form, i.e., the single time-step unitary operator $U$ admits a bilayer representation, where the local unitaries map a product of nearest-neighbor Hilbert spaces into another, with possibly different individual input or output dimensions.

Otherwise, the dynamics is said to be anomalous. As a natural application, this theory led to the discovery of new dynamical topological phases in 2D Floquet MBL systems [4-8], including the case of protecting symmetries [19-21], which go beyond the cohomology paradigm [22-24].

Although the index was initially defined in terms of abstract operator algebras [11], an equivalent definition, which reflects an intuitive picture of quantum-information 
flow $[7,17,18]$, was recently put forward. In turn, this made it possible to establish a lower bound on quantum scrambling in terms of the index, building a bridge between genuinely dynamical topological invariants and quantum chaos [17].

In this Letter, we develop a connection of a different kind: whereas Ref. [17] derived universal relations involving the index at the microscopic level, here we reveal its implications for the dynamics of generic systems at macroscopic (hydrodynamic) scales. This is done within the framework of the entanglement membrane theory (EMT) developed in Refs. [25-27]. Following the logic of these works, where random unitary circuit (RUC) models played a key role, our approach is based on the analysis of random QCA, which we propose as minimal models for anomalous chaotic systems.

Conventional EMT. - Let us begin by recalling the basic aspects of the EMT $[25,26]$. Throughout this Letter, we consider a 1D lattice of qudits associated with a Hilbert space $\left(\mathbb{C}^{d}\right)^{\otimes 2 L}(2 L$ : system size) and a unitary dynamics dictated by the operator $\mathcal{U}(t):\left(\mathbb{C}^{d}\right)^{\otimes 2 L} \rightarrow\left(\mathbb{C}^{d}\right)^{\otimes 2 L}$, where time $t$ might be either continuous or discrete.

The main object of the EMT is the so-called membrane tension (or line tension, in 1D), which associates an entanglement cost with a given spacetime cut through the unitary operator $\mathcal{U}(t)$ (cf. Fig. 2). This quantity allows for an intuitive geometric picture for the coarse-grained entanglement dynamics. The local tension $\mathcal{E}(v)$ is a function of the curve velocity $v=d x / d t$, and the cost of a given curve is obtained by integrating $\mathcal{E}(v)$ along its length. Then, the entanglement of a given interval $A$ in space at a given time is obtained by minimizing the integral of $\mathcal{E}(v)$ over all curves that separate a spacetime region that terminates on $A$ on the temporal boundary. As an example, we may consider the growth of the entanglement after a quench, for an infinite bipartite system with open boundary conditions: assuming homogeneous spacetime dynamics, we obtain

$$
S(x, t)=\min _{y}\left[t s_{\mathrm{eq}} \mathcal{E}\left(\frac{x-y}{t}\right)+S(y, 0)\right],
$$

where $S(y, 0)$ is the entanglement of the initial state, while $s_{\mathrm{eq}}$ is the entanglement density reached at equilibrium [26]. $S(x, t)$ here may indicate the von Neumann entanglement entropy or (assuming the absence of conservation laws [2831]) an arbitrary Rényi entropy [32]. Holographic field theories give elegant examples of off-lattice systems where $\mathcal{E}(v)$ is explicitly computable [33-35].

The EMT may be equivalently formulated in terms of a local entanglement production rate. In the bipartite setting above, the membrane picture is equivalent to a dynamical equation $\partial S / \partial t=s_{\mathrm{eq}} \Gamma(\partial S / \partial x)$, where $\Gamma(s)$ is a local production rate dependent on the entanglement gradient $[25,26]$. Comparison with (1) reveals that $\Gamma(s)$ and $\mathcal{E}(v)$ are simply related by the Legendre transformation (a) $t$
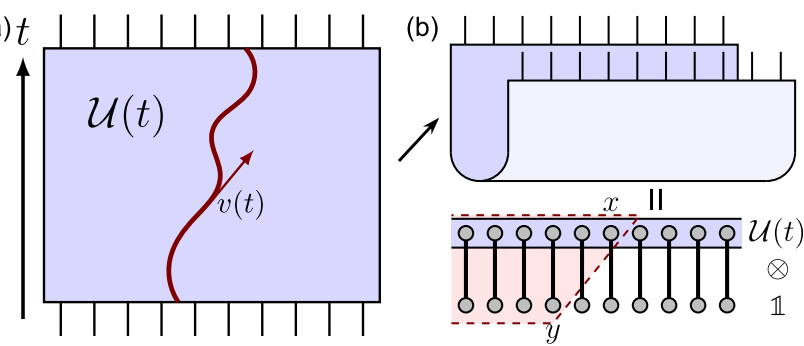

FIG. 2. (a) Pictorial representation of a spacetime curve cutting through the unitary evolution operator $\mathcal{U}(t)$, in $(1+1) \mathrm{D}$. For a given curve, the total line tension is $\int s_{\mathrm{eq}} \mathcal{E}(v) d t$, where $v(t)$ is the local velocity. (b) The line tension may be obtained by viewing $\mathcal{U}(t)$ as a state, and computing the corresponding bipartite entanglement.

$$
\Gamma(s)=\min _{v}\left[\mathcal{E}(v)-\frac{v s}{s_{\mathrm{eq}}}\right]
$$

The line tension also encodes information about the growth of local operators and, in general, must satisfy some basic constraints [26]. First, internal consistency of the coarse-grained picture requires that $\mathcal{E}(v) \geq 0$ and $\mathcal{E}^{\prime \prime}(v) \geq 0$. Second, one may argue that the minimization in Eq. (2) only involves membrane velocities within a range $\left[-v_{-}, v_{+}\right]$, where $v_{ \pm}$coincide with the left (right) butterfly speeds $v_{L, R}$ that govern the growth of local operators. Although $v_{L}=v_{R}$ if spatial inversion symmetry is present, this is not generally true otherwise, even for localHamiltonian dynamics [36-38]. The minimum of $\mathcal{E}(v)$ is the "entanglement velocity" $v_{E}$, quantifying the entanglement growth rate (rescaled by $s_{\mathrm{eq}}$ ) starting from a product state. Finally, one can show

$$
\mathcal{E}\left(v_{R}\right)=v_{R}, \quad \mathcal{E}\left(-v_{L}\right)=v_{L},
$$

and $\mathcal{E}^{\prime}\left(v_{R}\right)=-\mathcal{E}^{\prime}\left(-v_{L}\right)=1$, implying $v_{E} \leq \min \left\{v_{L}, v_{R}\right\}$. This picture is believed to hold for generic localHamiltonian and quantum-circuit evolution; our goal is to find whether and how it can be extended to anomalous dynamics.

The Margolus form for QCA.-As mentioned, the edge dynamics of the 2D systems in Fig. 1(a) is LP. This means that the single time-step unitary operator $U$ of the discrete evolution has the following property: for any local observable $O_{j}$ acting on site $j$, the operator $U^{\dagger} O_{j} U$ is supported on a finite neighborhood of $j$, up to exponentially decaying tails [39].

Any LP dynamics may be approximated arbitrarily well by QCA, by "chopping off" the exponential tails [18]. In turn, it is known that any QCA may be expressed in the socalled Margolus form [40] [cf. Fig. 1(b)], where $U$ is written as a two-layer product of two-site unitaries. This does not always define a quantum circuit because the dimensions of the local spaces associated with the "virtual" 
layer may differ from the physical ones: denoting by $p$ and $q$ two integers such that $d^{2}=p q$, we have

$$
U=\left(\otimes_{j=1}^{L} v_{2 j-1,2 j}\right)\left(\otimes_{j=1}^{L} u_{2 j, 2 j+1}\right),
$$

where $u: \mathbb{C}^{d} \otimes \mathbb{C}^{d} \rightarrow \mathbb{C}^{p} \otimes \mathbb{C}^{q}$ and $v: \mathbb{C}^{q} \otimes \mathbb{C}^{p} \rightarrow \mathbb{C}^{d} \otimes \mathbb{C}^{d}$. Given this representation, the topological index reads [11]

$$
\text { ind }=\frac{1}{2} \ln \frac{q}{p} \text {. }
$$

The unitary operation of translation by one site is a simple example with ind $=\ln d$. Note that for finite systems ind $\neq 0$ is only possible for periodic boundary conditions [41]. For simplicity, we will always take $L \rightarrow \infty$, so that the boundary conditions become irrelevant.

The Margolus representation allows us to pinpoint the essential feature of anomalous dynamics that we have to take into account in order to generalize the conventional EMT. First, we note that the QCA in Fig. 1(b) can be viewed as a unitary tensor network (TN) and that, although the dimensions associated with given bonds may vary in space and time, unitarity requires that the input and output dimensions of each tensor must match. This gives rise to a nontrivial local conservation law, not accounted for in conventional EMT.

Physically, we can understand such local conservation law as a continuity equation of the form $\partial_{\mu} J^{\mu}=0$, in terms of a coarse-grained spacetime entropy current $J^{\mu}$. For a unitary TN dynamics locally equilibrating to infinite temperature, $J^{\mu}$ has an explicit microscopic definition: Regarding the TN as a graph whose nodes are the unitaries and edges are the bonds, we orient the latter in the direction of increasing time; then, along each bond we define the entropy current as a vector in the direction of its orientation, whose magnitude is equal to $\ln d_{i}$, where $d_{i}$ ia the associated local Hilbert-space dimension.

The coarse-grained spacetime entropy current is more general than the model above, where it can be introduced by "counting" microscopic bonds. For instance, it can be defined even when the equilibrium state is nontrivial and determined by the slow modes [42]. In any case, it has important consequences on the properties of the membrane tension. In the following, we show how the EMT has to be modified in the presence of a nontrivial spacetime entropy current. The resulting generalized EMT turns out to correctly capture the coarse-grained features of anomalous dynamics, finally revealing the hydrodynamic implications of the index.

Generalized EMT.-As a starting point to generalize the EMT, we assume there is a well-defined line tension $\mathcal{E}(v)$ satisfying $\mathcal{E}(v), \mathcal{E}^{\prime \prime}(v) \geq 0$ (as required for consistency of the hydrodynamic picture). We also postulate that there exists a spacetime entropy current $J^{\mu}(x, t)=\left(J_{x}, J_{t}\right)$ that governs the growth and transport of thermodynamic entropy. In particular, the density $s$ of thermodynamic entropy (we assume local equilibrium) is equal to $J_{t}$.

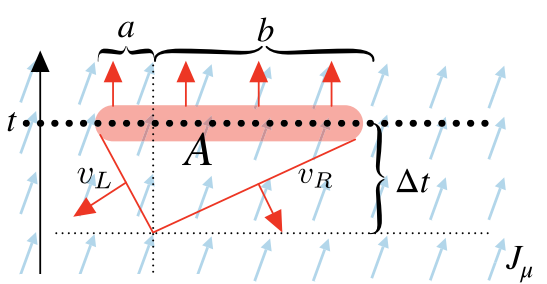

FIG. 3. In the stationary regime, the divergence of the entropy current is vanishing, and the flux through any closed surface is zero. In order to compute the integral (6), we choose a surface containing the optimal membranes and exploit the prescriptions of the EMT.

Let us consider a stationary regime, focusing, for instance, on a finite interval $A$ at large times after a quench. Stationarity requires $\partial_{\mu} J^{\mu}=0$ and also implies that the thermodynamic entropy equals the von Neumann entropy, $S_{A}(t)$, i.e.,

$$
S_{A}(t)=\int_{A} d x J_{t}(x)
$$

Using the divergence theorem, the integral over $A$ may be obtained by integrating the current over any closed perimeter containing $A$ (cf. Fig. 3 ). In order to make contact with the EMT, we choose the perimeter to be a triangle whose bottom sides have slopes given by the butterfly velocities $-v_{L}$ and $v_{R}$. A simple computation then yields $S_{A}(t)=$ $\left(J_{x} \Delta t+J_{t} a\right)+\left(-J_{x} \Delta t+J_{t} b\right)$, where $\Delta t, a$, and $b$ are as in Fig. 3. On the other hand, the sides of triangle minimize the line tension for the region $A$, as one can show by generalizing the arguments of [26]. As a consequence, $S_{A}(t)=s_{\text {eq }}\left[\mathcal{E}\left(-v_{L}\right) \Delta t+\mathcal{E}\left(v_{R}\right) \Delta t\right]$. Identifying the individual terms coming from the two bottom sides of the triangle, and using $a / \Delta t=v_{L}$ and $b / \Delta t=v_{R}$, we find $J_{t}=\left[s_{\mathrm{eq}} /\left(v_{L}+v_{R}\right)\right]\left[\mathcal{E}\left(-v_{L}\right)+\mathcal{E}\left(v_{R}\right)\right]$ and $J_{x}=\left[s_{\text {eq }} /\left(v_{L}+\right.\right.$ $\left.\left.v_{R}\right)\right]\left[v_{R} \mathcal{E}\left(-v_{L}\right)-v_{L} \mathcal{E}\left(v_{R}\right)\right]$. Defining now the "background entropy velocity" $v^{*}=J_{x} / J_{t}$ and using $S_{A}(t)=$ $s_{\text {eq }}(a+b)$ (which follows from stationarity), we finally obtain

$$
\mathcal{E}\left(v_{R}\right)=v_{R}-v^{*}, \quad \mathcal{E}\left(-v_{L}\right)=v_{L}+v^{*} .
$$

This equation deviates from the conventional EMT, cf. (3), and has important ramifications. In particular, combined with convexity, it implies $\mathcal{E}(v) \geq\left|v-v^{*}\right|$ and so $v_{E} \leq$ $\min \left\{v_{R}-v^{*}, v_{L}+v^{*}\right\}$.

Using (7), one can also argue that the relation between $\Gamma(s)$ and $\mathcal{E}(v)$ must be modified. Indeed, plugging (7) directly into (2), we see that $\Gamma(s)<0$ for some values of $s$. This is clearly an inconsistency, since $\Gamma(s)$ is the rate of entanglement growth. In order to guarantee positivity, one is led to the natural generalization

$$
\Gamma(s)=\min _{v}\left[\mathcal{E}(v)-\frac{v s}{s_{\mathrm{eq}}}\right]+v^{*} \frac{s}{s_{\mathrm{eq}}} .
$$


From elementary properties of the Legendre transformation, we have the basic constraints $-s_{\mathrm{eq}} \Gamma^{\prime}\left(s_{\mathrm{eq}}\right)=v_{R}-v^{*}$, $s_{\text {eq }} \Gamma^{\prime}\left(-s_{\text {eq }}\right)=v_{L}+v^{*}$. Note that by construction $\Gamma(0)=$ $v_{E}$. Finally, differentiating (1) with respect to $t$, we obtain

$$
\frac{\partial S}{\partial t}+v^{*} \frac{\partial S}{\partial x}=s_{\mathrm{eq}} \Gamma\left(\frac{\partial S}{\partial x}\right) .
$$

We see that the fundamental equation from the EMT, governing the coarse-grained entanglement dynamics, is modified by a constant velocity term. Importantly, $v^{*}$ is now left as a free parameter, and the conventional EMT is recovered for $v^{*}=0$. Note that without entropy production, i.e., $\Gamma(\partial S / \partial x)=0$, Eq. (9) still predicts a nonzero entropy change, which is qualitatively different from normal dynamics.

Models of random QCA.- - In order to test the generalized EMT and identify the entropy-current velocity $v^{*}$, we study concrete models of chaotic anomalous dynamics. We consider QCA of the form (4) where $u$ and $v$ at different spacetime positions are drawn independently from the Haar random ensemble. Generalizing from the special case of RUCs, we expect the model also to capture universal aspects of random Floquet evolutions [43-49] and translationally invariant homogeneous systems [25-27,50] if we restrict to the leading dynamics at large scales. We note that it is easy to construct explicit 2D models with trivial bulk dynamics that display the random edge evolution considered here. This construction is detailed in the Supplemental Material, where we also define even simpler random 1D QCA that appear naturally in this context [51].

As a first step, we analyze how the support of a localized traceless operator $O_{0}$ grows under the dynamics, which allows us to extract the butterfly velocities. We focus on the out-of-time-order correlator (OTOC) $\mathcal{C}(x, t)=$ $\operatorname{tr}\left\{\left[O_{0}, O_{2 x}^{\prime}(t)\right]^{\dagger}\left[O_{0}, O_{2 x}^{\prime}(t)\right]\right\} / 2$ [57-61]. Here $O_{2 x}^{\prime}(t)=$ $\mathcal{U}(t)^{\dagger} O_{2 x}^{\prime} \mathcal{U}(t)$ and $O_{2 x}^{\prime}$ is a traceless operator supported at site $2 x$. Given the brickwork structure of the random QCA, the disorder-averaged OTOC $\overline{\mathcal{C}(x, t)}$ may be computed using the approach developed for RUCs, mapping the problem to the partition function of an Ising-like model [62,63], see also [64-69]. Additional technical complications arise due to the "staggered" structure of the dynamics, alternating physical and virtual Hilbert spaces [cf. Fig. 1(b)]. Nevertheless, a fully analytic expression may be obtained [51], and in the hydrodynamic limit of large spacetime scales, it simply reads $\overline{\mathcal{C}(x, t)} \simeq \Phi\left(\left(v_{L} t+x\right) / \sigma_{L}\right) \Phi\left(\left(v_{R} t-\right.\right.$ $\left.x) / \sigma_{R}\right)$. Here $\Phi(y)=(2 \pi)^{-1 / 2} \int_{-\infty}^{y} e^{-x^{2} / 2} d x$ and

$$
\begin{gathered}
v_{L}=\frac{p^{2} q^{2}-q(p+q)+1}{p^{2} q^{2}-1}, \\
\sigma_{L}=\frac{\sqrt{t} \sqrt{q\left[p^{3} q^{2}+p^{2} q\left(q^{2}-3\right)+p-q^{3}+q\right]}}{p^{2} q^{2}-1},
\end{gathered}
$$

while $v_{R}$ and $\sigma_{R}$ are obtained by exchanging $p \leftrightarrow q$. We see that the coarse-grained OTOC has the same form as RUCs
$[62,63]$, being characterized by two propagating fronts inside which $\overline{\mathcal{C}(x, t)} \simeq 1$; i.e., information is fully scrambled. However, the fronts propagate with asymmetric butterfly velocities and widths, with the faster front being the narrowest one (in contrast to the quantum-circuit models constructed in Ref. [37]). Note that, when ind $=0$, i.e., $p=q$, we recover the result for RUCs $[62,63]$. In the other limit, where ind $=\ln d$, i.e., $p=1$ and $q=d^{2}$, the random QCA consists of a two-step evolution in which a right translation is followed by a layer of random unitary gates. Since the shift does not increase the operator support, $\sigma_{L, R}$ in (10) are then simply those of a RUC evolved up to a time $t^{\prime}=t / 2$.

Entanglement dynamics. - Next, we move on to compute the line tension, cf. Fig. 2. Formally, we introduce a doubled Hilbert space $\mathcal{H}=\left(\mathbb{C}^{d}\right)^{\otimes 2 L} \otimes\left(\mathbb{C}^{d}\right)^{\otimes 2 L}$ along with the maximally entangled state $|I\rangle=\left|\phi_{+}\right\rangle^{\otimes 2 L}$, where $\left|\phi_{+}\right\rangle \equiv \sum_{n=1}^{d}|n\rangle \otimes|n\rangle / \sqrt{d}$ and $n$ runs over a basis of $\mathbb{C}^{d}$. This allows us to vectorize the evolution operator as $|\mathcal{U}(t)\rangle=[\mathcal{U}(t) \otimes \mathbb{1}]|I\rangle \in \mathcal{H}$, with the two Hilbert spaces associated with its input and output degrees of freedom. Considering a bipartition of the system with boundary at site $x$ and $y$ in the input and output, respectively, we define the operator entanglement [70-73] $S^{(\mathrm{o})}(y-x, t)$ as the associated entanglement entropy of $|\mathcal{U}(t)\rangle$. The line tension may then be computed via [26] $\mathcal{E}(v)=\lim _{t \rightarrow \infty} S^{(o)}(v t, t) /\left(s_{\text {eq }} t\right)$, for $v$ in the range $\left(-v_{-}, v_{+}\right)$. Our random QCA has no conserved quantity and our unit length increment $d x=1$ is defined to contain two sites, so that $s_{\mathrm{eq}}=\ln d^{2}=\ln (p q)$. $v^{(o)}=\mathcal{E}(0)$ is the operator-entanglement velocity.

Averaged Rényi- $n$ entropies are hard to compute, but the mapping to an Ising partition function gives access to the averaged purity and its logarithm $-\ln \overline{e^{-S_{2}^{(o)}(x, t)}}$. Since this average is taken "inside the logarithm," it differs from the averaged Rényi-2 entropy $\overline{S_{2}^{(\mathrm{o})}}$. However, the former is sufficient to see the key relationships obeyed by $\mathcal{E}$. The line tension for $\overline{S_{2}^{(0)}}$ can be understood as a perturbatively "dressed" version of that for $-\ln \overline{e^{-S_{2}^{(0)}(x, t)}}$ [27]: the difference between the two vanishes as $d \rightarrow \infty$ and, in fact, is numerically small even for finite $d$ [74]. So we approximate $t^{-1} \overline{S_{2}^{(\mathrm{o})}(x, t)} \simeq-t^{-1} \ln \overline{e^{-S_{2}^{(o)}(x, t)}}$, yielding [51]

$$
\begin{aligned}
\mathcal{E}_{2}(v)= & \log _{d^{2}} \frac{(p q+1)^{2}(p q-1)}{p q \sqrt{\left(p^{2}-1\right)\left(q^{2}-1\right)}} \\
& -v \log _{d^{2}} \frac{\sqrt{\left(1-r_{p}\right)^{2} v^{2}+4 r_{p}}-\left(1+r_{p}\right) v}{2(1+v)} \\
& -\log _{d^{2}} \frac{1+r_{p}+\sqrt{\left(1-r_{p}\right)^{2} v^{2}+4 r_{p}}}{\left(1-v^{2}\right) \sqrt{r_{p}}},
\end{aligned}
$$



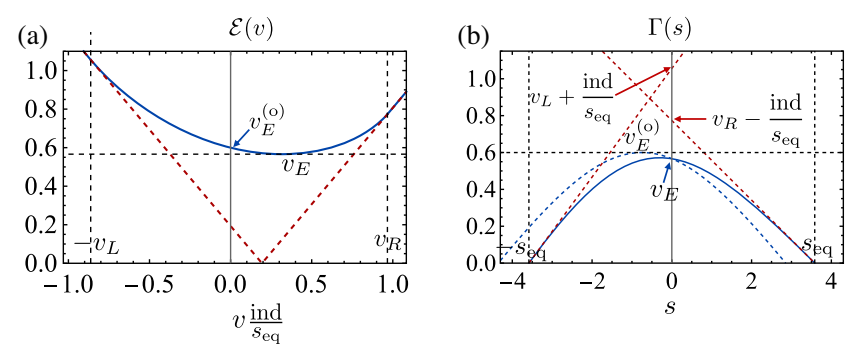

FIG. 4. (a) Entanglement line tension $\mathcal{E}(v)$ and (b) production $\Gamma(s)$ (in terms of Rényi-2 entropy) for the random QCA model with $d=6$ and $p=3, q=12$. The red dashed lines refer to the tangents at (a) $\left(v_{L, R}, \mathcal{E}\left(\mp v_{L, R}\right)=v_{L, R} \pm \mathrm{ind} / s_{\text {eq }}\right)$; (b) $\left( \pm s_{\text {eq }}, 0\right)$. The blue dashed line in (b) corresponds to $\Gamma(s)-$ (ind $\left./ s_{\text {eq }}\right)\left(s / s_{\text {eq }}\right)$, whose maximum gives $v_{E}^{(\text {o) }}$.

where $r_{p}=p\left(q^{2}-1\right) /\left[q\left(p^{2}-1\right)\right]$. As a main difference from the case of RUCs, the minimum is at a nonzero velocity $v_{m}=\left(r_{p}-1\right) /\left[2\left(r_{p}+1\right)\right]$ [cf. Fig. 4(a)]. The value $\mathcal{E}_{2}\left(v_{m}\right)$ yields the entanglement speed $v_{E}=$ $\log _{d^{2}}\left\{(p q+1)^{2} /[2 \sqrt{p q}(p+q)]\right\}$, as confirmed by directly computing the growth of state Rényi-2 entropy following a quench from a product state [51]. We stress that $v_{L, R}$ and $v_{E}$ do not depend on ind in a universal way. This could be expected from the study of RUCs, where asymmetric butterfly velocities might be realized by specific arrangements of the local unitaries [37].

Crucially, we see that $\mathcal{E}_{2}^{\prime}\left( \pm v_{ \pm}\right)= \pm 1$, where $v_{ \pm}=v_{R, L}$, and that Eq. (7) is satisfied, after the identification

$$
v^{*}=\frac{\text { ind }}{s_{\text {eq }}} .
$$

This is our final main result: it states that the index, a microscopic dynamical topological invariant, appears at the hydrodynamic level as a constant background velocity for the coarse-grained entanglement dynamics. Based on this identification, the index also determines the qualitative features of the rate $\Gamma(s)$, which is shown in Fig. 4. Further details on the random QCA, including a computation of the so-called tripartite mutual information [75] and its relation to the index, are reported in the Supplemental Material [51].

Outlook.-Our results open up several possibilities for future research. First, when viewing anomalous 1D dynamics as boundaries of 2D Floquet systems, it would be interesting to investigate the corrections to our theory when the assumption of ideal localization in the bulk is relaxed. In this case, we expect subleading effects emerging, due to a slow entropy flow from the boundary to the bulk and vice versa. It would also be natural to apply our picture based on spacetime entropy currents to more general situations with inhomogeneous backgrounds, as models with genuinely spacetime-dependent entropy currents may be constructed by introducing additional structure. Next, it would be interesting to explore how chaotic anomalous dynamics is modified by local conservation laws, such as $\mathrm{U}(1)$ charges as done for RUCs [64,65]. Studies along this direction could reveal an intriguing effect of the index on the otherwise purely diffusive behavior of the charge. Finally, two natural generalizations of our study include adding randomized measurements [76-83] and higher dimensions, where the theory of QCA is much more open [84-87].

We acknowledge Ignacio Cirac and David Huse for helpful discussions. We thank the anonymous referee for valuable comments on the Letter. Z. G. is supported by the Max-Planck-Harvard Research Center for Quantum Optics (MPHQ).

[1] E. Lieb and D. Robinson, Commun. Math. Phys. 28, 251 (1972).

[2] P. Arrighi, Nat. Comput. 18, 885 (2019).

[3] T. Farrelly, Quantum 4, 368 (2020).

[4] H. C. Po, L. Fidkowski, T. Morimoto, A. C. Potter, and A. Vishwanath, Phys. Rev. X 6, 041070 (2016).

[5] H. C. Po, L. Fidkowski, A. Vishwanath, and A. C. Potter, Phys. Rev. B 96, 245116 (2017).

[6] F. Harper and R. Roy, Phys. Rev. Lett. 118, 115301 (2017).

[7] B. R. Duschatko, P. T. Dumitrescu, and A. C. Potter, Phys. Rev. B 98, 054309 (2018).

[8] L. Fidkowski, H. C. Po, A. C. Potter, and A. Vishwanath, Phys. Rev. B 99, 085115 (2019).

[9] To be more precise, MBL is believed to be unstable in two and higher dimensions (i.e., strict MBL requires fine-tuning of the dynamics). However, localization can persist for times double-exponentially long in disorder strength, so for practical purposes can resemble a phase (see Ref. [4] for discussion in the QCA context).

[10] P. Arrighi, V. Nesme, and R. Werner, J. Comput. Syst. Sci. 77, 372 (2011).

[11] D. Gross, V. Nesme, H. Vogts, and R. F. Werner, Commun. Math. Phys. 310, 419 (2012).

[12] T. C. Farrelly and A. J. Short, Phys. Rev. A 89, 012302 (2014).

[13] J. I. Cirac, D. Perez-Garcia, N. Schuch, and F. Verstraete, J. Stat. Mech. (2017) 083105.

[14] M. B. Sahinoglu, S. K. Shukla, F. Bi, and X. Chen, Phys. Rev. B 98, 245122 (2018).

[15] L. Piroli and J. I. Cirac, Phys. Rev. Lett. 125, 190402 (2020).

[16] L. Piroli, A. Turzillo, S. K. Shukla, and J. I. Cirac, J. Stat. Mech. (2021) 013107.

[17] Z. Gong, L. Piroli, and J. I. Cirac, Phys. Rev. Lett. 126, 160601 (2021).

[18] D. Ranard, M. Walter, and F. Witteveen, arXiv:2012.00741.

[19] Z. Gong, C. Sünderhauf, N. Schuch, and J. I. Cirac, Phys. Rev. Lett. 124, 100402 (2020).

[20] C. Zhang and M. Levin, Phys. Rev. B 103, 064302 (2021).

[21] Z. Gong and T. Guaita, arXiv:2106.05044.

[22] D. V. Else and C. Nayak, Phys. Rev. B 93, 201103(R) (2016). 
[23] A. C. Potter and T. Morimoto, Phys. Rev. B 95, 155126 (2017).

[24] R. Roy and F. Harper, Phys. Rev. B 95, 195128 (2017).

[25] A. Nahum, J. Ruhman, S. Vijay, and J. Haah, Phys. Rev. X 7, 031016 (2017).

[26] C. Jonay, D. A. Huse, and A. Nahum, arXiv:1803.00089.

[27] T. Zhou and A. Nahum, Phys. Rev. X 10, 031066 (2020).

[28] T. Rakovszky, F. Pollmann, and C. W. von Keyserlingk, Phys. Rev. Lett. 122, 250602 (2019).

[29] Y. Huang, IOP SciNotes 1, 035205 (2020).

[30] T. Rakovszky, C. W. von Keyserlingk, and F. Pollmann, Phys. Rev. B 100, 125139 (2019).

[31] T. Zhou and A. W. W. Ludwig, Phys. Rev. Research 2, 033020 (2020).

[32] M. A. Nielsen and I. Chuang, Quantum Computation and Quantum Information (Cambridge University Press, Cambridge, England, 2002).

[33] M. Mezei, Phys. Rev. D 98, 106025 (2018).

[34] M. Mezei and J. Virrueta, J. High Energy Phys. 02 (2020) 013.

[35] C. A. Agón and M. Mezei, J. High Energy Phys. 11 (2021) 167.

[36] F. Liu, J. R. Garrison, D.-L. Deng, Z.-X. Gong, and A. V. Gorshkov, Phys. Rev. Lett. 121, 250404 (2018).

[37] C. Stahl, V. Khemani, and D. A. Huse, arXiv:1812.05589.

[38] Y.-L. Zhang and V. Khemani, SciPost Phys. 9, 24 (2020).

[39] The index theory is, in fact, robust even when locality is preserved up to polynomially decaying tails [18]. However, analogous to the case of local-Hamiltonian evolution, longrange tails might change some qualitative features of the dynamics and are not considered in this Letter.

[40] More precisely, any QCA may be represented in this way, up to grouping together finite sets of neighboring sites [3].

[41] More precisely, a nonzero index is incompatible with a static spatial boundary that preserves unitarity. A unitary boundary moving at coarse-grained velocity $v^{*}$ [defined in (12)] can be constructed, as seen most easily when $U$ is the translation operator with $v^{*}=1$.

[42] Details will be provided in future works.

[43] A. Chan, A. De Luca, and J. T. Chalker, Phys. Rev. X 8, 041019 (2018).

[44] A. Chan, A. De Luca, and J. T. Chalker, Phys. Rev. Lett. 121, 060601 (2018).

[45] B. Bertini, P. Kos, and T. Prosen, Phys. Rev. Lett. 121, 264101 (2018).

[46] C. Sünderhauf, D. Pérez-García, D. A. Huse, N. Schuch, and J. I. Cirac, Phys. Rev. B 98, 134204 (2018).

[47] B. Bertini, P. Kos, and T. Prosen, Phys. Rev. X 9, 021033 (2019).

[48] A. J. Friedman, A. Chan, A. De Luca, and J. T. Chalker, Phys. Rev. Lett. 123, 210603 (2019).

[49] A. Chan, A. De Luca, and J. T. Chalker, Phys. Rev. Lett. 122, 220601 (2019).

[50] S. J. Garratt and J. T. Chalker, Phys. Rev. X 11, 021051 (2021).

[51] See Supplemental Material at http://link.aps.org/ supplemental/10.1103/PhysRevLett.128.080602 for further details, which includes Refs. [52-56].

[52] R. B. Paris, J. Class. Anal. 2, 183 (2013).

[53] R. B. Paris, J. Class. Anal. 3, 1 (2013).
[54] C. Sünderhauf, L. Piroli, X.-L. Qi, N. Schuch, and J. I. Cirac, J. High Energy Phys. 11 (2019) 038.

[55] O. Schnaack, N. Bölter, S. Paeckel, S. R. Manmana, S. Kehrein, and M. Schmitt, Phys. Rev. B 100, 224302 (2019).

[56] J. Kudler-Flam, R. Sohal, and L. Nie, arXiv:2107.04043.

[57] A. Larkin and Y. N. Ovchinnikov, Sov Phys JETP 28, 1200 (1969), http://www.jetp.ras.ru/cgi-bin/dn/e_028_06_1200 .pdf.

[58] A. Kitaev, Hidden correlations in the Hawking radiation and thermal noise, In The Fundamental Physics Prize Symposium (2014).

[59] S. H. Shenker and D. Stanford, J. High Energy Phys. 03 (2014) 067.

[60] S. H. Shenker and D. Stanford, J. High Energy Phys. 12 (2014) 046.

[61] J. Maldacena, S. H. Shenker, and D. Stanford, J. High Energy Phys. 08 (2016) 106.

[62] A. Nahum, S. Vijay, and J. Haah, Phys. Rev. X 8, 021014 (2018).

[63] C. W. von Keyserlingk, T. Rakovszky, F. Pollmann, and S. L. Sondhi, Phys. Rev. X 8, 021013 (2018).

[64] V. Khemani, A. Vishwanath, and D. A. Huse, Phys. Rev. X 8, 031057 (2018).

[65] T. Rakovszky, F. Pollmann, and C. W. von Keyserlingk, Phys. Rev. X 8, 031058 (2018).

[66] N. Hunter-Jones, arXiv:1812.08219.

[67] N. Hunter-Jones, arXiv:1905.12053.

[68] T. Zhou and A. Nahum, Phys. Rev. B 99, 174205 (2019).

[69] B. Bertini and L. Piroli, Phys. Rev. B 102, 064305 (2020).

[70] P. Zanardi, Phys. Rev. A 63, 040304(R) (2001).

[71] T. Prosen and I. Pižorn, Phys. Rev. A 76, 032316 (2007).

[72] J. Dubail, J. Phys. A 50, 234001 (2017).

[73] T. Zhou and D. J. Luitz, Phys. Rev. B 95, 094206 (2017).

[74] For RUCs, it was proven that the two quantities coincide up to order $1 /\left(d^{8} \ln d\right)$ [68].

[75] P. Hosur, X.-L. Qi, D. A. Roberts, and B. Yoshida, J. High Energy Phys. 02 (2016) 004.

[76] Y. Li, X. Chen, and M. P. A. Fisher, Phys. Rev. B 98, 205136 (2018).

[77] B. Skinner, J. Ruhman, and A. Nahum, Phys. Rev. X 9, 031009 (2019).

[78] A. Chan, R. M. Nandkishore, M. Pretko, and G. Smith, Phys. Rev. B 99, 224307 (2019).

[79] R. Fan, S. Vijay, A. Vishwanath, and Y.-Z. You, Phys. Rev. B 103, 174309 (2021).

[80] S. Choi, Y. Bao, X.-L. Qi, and E. Altman, Phys. Rev. Lett. 125, 030505 (2020)

[81] M. J. Gullans and D. A. Huse, Phys. Rev. X 10, 041020 (2020).

[82] M. Ippoliti, M. J. Gullans, S. Gopalakrishnan, D. A. Huse, and V. Khemani, Phys. Rev. X 11, 011030 (2021).

[83] C.-M. Jian, Y.-Z. You, R. Vasseur, and A. W. W. Ludwig, Phys. Rev. B 101, 104302 (2020).

[84] J. Haah, L. Fidkowski, and M. B. Hastings, arXiv: 1812.01625 .

[85] J. Haah, J. Math. Phys. (N.Y.) 62, 092202 (2021).

[86] M. Freedman, J. Haah, and M. B. Hastings, arXiv:1910.07998.

[87] M. Freedman and M. B. Hastings, Commun. Math. Phys. 376, 1171 (2020). 